\title{
Impact of Inter-eNodeB Handover Parameters on Performance Optimization of LTE Networks
}

\author{
Mina Malekzadeh, Fatemeh Rezaiee \\ Faculty of Electrical and Computer Engineering, Hakim Sabzevari University Sabzevar, Iran
}

\begin{tabular}{l}
\hline \hline Article Info \\
\hline Article history: \\
Received Aug 27, 2017 \\
Revised Nov 5, 2017 \\
Accepted Nov 24, 2017 \\
\hline
\end{tabular}

\section{Keywords:}

Handover parameters

LTE mobility

LTE networks

Measurement events

\begin{abstract}
Handover, as a resource consuming process, is an important issue in mobile communications which can highly affect the overall performance of the LTE networks. Since the handover process mainly relies on four main entry condition parameters, optimizing these parameters will in turn improve the handover process. In this work, we investigate the effectiveness of the four handover parameters by measuring the resulting downlink throughput and number of the required handovers. We furthermore determine the optimized values of the parameters through implementing different sets of experiments. Finally, by applying the obtained optimized parameters on two main LTE handover algorithms i.e. A3RSRP and A2A4RSRQ, their performance is compared regarding to different speeds of the mobile equipment. We determine how to accurately select the optimal settings for the parameters so that greater LTE performance can be achieved.
\end{abstract}

Copyright $@ 2018$ Institute of Advanced Engineering and Science. All rights reserved.

\section{Corresponding Author:}

Mina Malekzadeh,

Department of Electrical and Computer Engineering,

Hakim Sabzevari University,

Sabzevar, Iran.

Email: m.malekzadeh@hsu.ac.ir

\section{INTRODUCTION}

The X2-based handover [1], happens when the mobile user equipment (UE) moves inter the eNodeBs, from the serving eNodeB (S-eNB) to the target eNodeB (T-eNB), without involving the evolved packet core (EPC) [2]. As the UE moves, the S-eNB keeps its track and decides whether a handover is required. This process involves measurements and decision procedure [3]. To make such a decision, a set of event-based measurements are performed by both UE and S-eNB.

The measurements performed by UE are Reference Signal Received Power (RSRP) and Reference Signal Received Quality (RSRQ) [4, 5]. The RSRP is a cell-specific signal strength related metric that is used as an input for the cell reselection and handover decisions. For a particular cell, the RSRP is defined as the average power (in $\mathrm{dBm}$ ) of the Resource Elements (RE) that carries cell-specific Reference Signals (RS) within the considered bandwidth. The RSRP measurement is utilized mainly to make ranking among different candidate cells in accordance with their signal strength.

The RSRQ measurement (in dB) is a cell-specific signal quality metric. Similar to the RSRP measurement, this metric is used mainly to provide ranking among different candidate cells but in accordance with their signal quality. This metric can be employed as an input in making cell reselection and handover decisions in scenarios in which the RSRP measurements are not sufficient to make reliable cell-reselection and handover decisions [5].

After measuring the RSRQ and RSRP, the conditions for any of the five predefined events are checked to examine if they are met. The LTE defines that the measurement reporting events as A1, A2, A3, A4 and A5 are based upon either RSRP or RSRQ messages. The events that can trigger the measurement report in X2-based handover are listed in Table 1. 
Table 1. LTE measurement events

\begin{tabular}{ll}
\hline Event & Description \\
\hline Event A1 & Serving becomes better than threshold \\
Event A2 & Serving becomes worse than $(\leq)$ threshold \\
Event A3 & Neighbor becomes an offset better than serving \\
Event A4 & Neighbor becomes better than threshold \\
Event A5 & Serving becomes worse than threshold1 and neighbor becomes better than threshold2 \\
\hline
\end{tabular}

When the required conditions for any of the five events are met, then a measurement report is triggered and sent back to the S-eNB in response to its measurement control message [6, 7]. Then, once the S-eNB makes a handover decision, it communicates with the T-eNB to inform it of the handover and also allocation of the required resources. After that, the UE detaches from the S-eNB and joins the T-eNB. To help the UEs accomplish reporting the measurement to the S-eNB, the overall handover process can be controlled by several parameters. The conditions of the events based the Table 1 show that the operation of the entire handover procedure mainly relies on the following four handover parameters [8]:

1. Threshold: refers to an absolute value to which the received signal level is compared with to determine whether it drops below or not.

2. Hysteresis: there can be a delay between when the UE decides to send a measurement report to initiate a handover until the handover actually happens. During this delay, due to frequent movement of the UE, the signal quality of the T-eNB may change for it and become weaker which will lead to a poor handover and the ping-pong effect [9]. Hysteresis is a parameter that is measured regarding to the RSRP and is used to make the current measured value of the T-eNB looks worse than it actually is. It helps the UE to assure that the T-eNB is strong enough at the time of triggering the handover.

3. Offset: is the parameter that makes the S-eNB looks better than its actual current measured value. As the offset can have either positive or negative values, it helps to accordingly speed up or slow down the handover process upon the requirements.

4. Time to trigger: depending on the network conditions, there can be quick variations in the signal quality of the T-eNB. In this case, in one hand the short-time peaks of the measured signals can satisfy the conditions to wrongly trigger the handover while on the other hand the quality of the signal is quickly dropped again and the conditions are not suitable to trigger the handover anymore. The Time-to-trigger (TTT) is a certain amount of time that UE waits after the handover condition is satisfied. Thus, the TTS like hysteresis delays the handover but regarding to the time not RSRP.

As we can see, in order to decide the handover moment, the handover parameters are directly involved in the handover decision making procedure. Thus accurately selecting the correct values for these parameters is a main challenge for optimization of the overall LTE performance. Wrong values for the parameters, either too high or too low, can lead to performance degradation as the result of performing either excessive number of handovers, too early or too late handovers, unnecessary handovers, or handover to the wrong T-eNB [10].

Thus, this work investigates the impact of the four handover parameters i.e. hysteresis, threshold, time to trigger, and offset and determine their individual optimal values under the current LTE handover algorithms i.e. A2A4-RSRQ and A3-RSRP, to optimize the LTE performance.

The rest of this work is organized as follows. Section 2 reviews the related works. Section 3 presents the simulation environment along with the corresponding scenarios. Section 4 analyzes the experimental results. The work is concluded in section 5 .

\section{RELATED WORKS}

Providing mobility and tracking the users as they move between different eNodeBs, is one of the major functions of the cellular networks including LTE. The LTE handover is a resource consuming procedure which can highly degrade the overall performance of the network if it does not perform precisely. On the other hand, existence of a variety of parameters involved in the handover including threshold, hysteresis, offset, and time to trigger, has added more complexity to the procedure. Thus it is essential to define an analytical model regarding to different values of different handover parameters to determine the optimal values that effectively enhance the handover procedure.

Due to significance of the issue, there are many researches that study the handover management. However, only a few of them take into account the optimization of the handover parameters. The authors in [11] use NS3 and consider the existing handover algorithms for LTE which are A2A4-RSRQ and A3-RSRP. They utilize the functionalities of both algorithms to provide analysis of different parameters including throughput, SINR, and number of handover. 
However, evaluation of the handover parameters is not included. The hysteresis margin in considered in A3 event in a heterogeneous network using LTE-sim tool [9].

The throughput, rate of radio link failure, and ping-pong effect are analyzed with different number of UEs without regard to the effectiveness of the handover parameters. The optimized handover parameters under the downlink LTE are provided in [12] using a new handover algorithm under a C++ platform. Different amounts of speed are considered for mobility of the UE. Through the results, the effectiveness of the time to trigger is discussed for the proposed model while the common handover algorithms are not evaluated.

In [13] a commercial measurement tool called NEMO Handy, is used for live capturing of the LTE traffic to verify the possible relationships among SINR, RSRP, RSSI and RSRQ as well as evaluating the effects of SNR on throughput. However, verification is not applied on the handover parameters. The optimization of time to trigger and hysteresis are considered in [14].

The speed of user is fixed at $120 \mathrm{~km} / \mathrm{h}$ to evaluate the A3 event while A2 and A4 events are not studied. The X2 handover is also studied in [15] using OpenAirInterface LTE emulation platform. The impact of the RSRP on the handover decision algorithm is investigated while the other handover parameters are not included. By varying user velocity, the authors in [16] investigate the impact of time to trigger on the A3 event. The handover algorithms are also investigated in $[17,18]$ along with LTE networks specifications in [19,20,21].

Considering the lack of investigating the impact of all four handover parameters on both handover algorithms in the related works, this work provides several simulated scenarios based on the four handover parameters i.e. threshold, hysteresis, offset, and time to trigger with both A2A4-RSRQ and A3-RSRP algorithms. The main contribution of this work is to precisely evaluate a variety of different values for the handover parameters for two existing handover algorithms. The objective is by providing a careful comparison, we determine the optimal values of the four handover parameters in terms of the following performance metrics:

1) Minimizing number of unnecessary handovers

2) Minimizing impact of handover on service performance in term of throughput

\section{SIMULATION SETUP}

As mentioned, the main purpose of this work is to find the optimal values of the handover parameters to enhance the handover procedure in early stages before it actually happens. In this section, we present the network environment that we develop along with the five corresponding scenarios.

\subsection{Simulation Environment}

The NS3 simulation tool is used to develop the LTE network with four eNodeBs and one SGW/PGW. Furthermore, a remote server, outside of the LTE network, is assigned as the end user to communicate with the UE in the LTE network through SGW/PGW. The UE is on the car which is moving around the network while handing over from one eNodeB to another Figure 1.

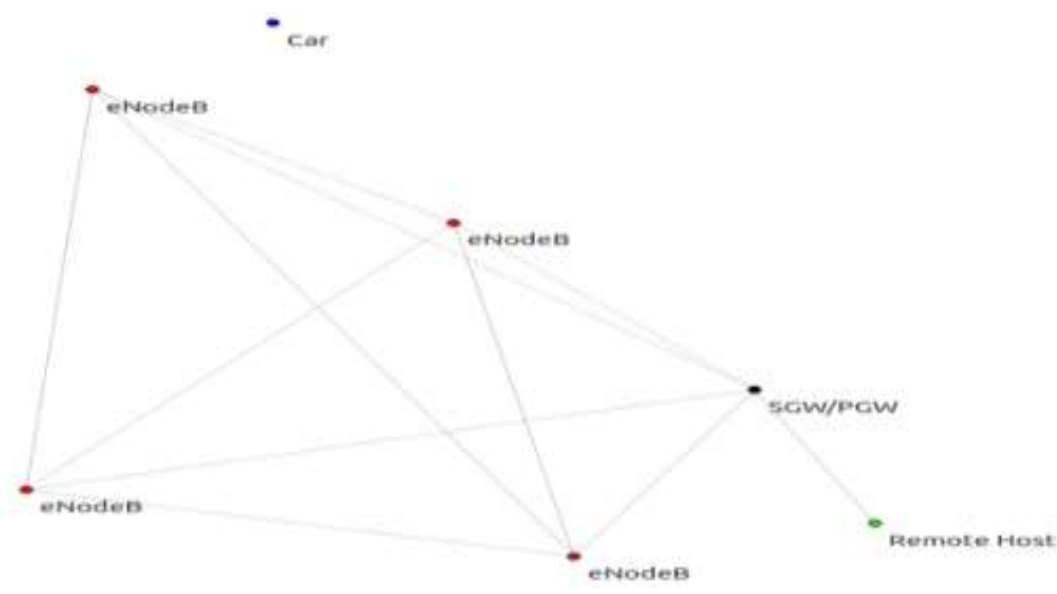

Figure 1. Simulation environment 
The location of the components existing in this topology are as follow:

1. eNodeB 1 is in $(200,300) \mathrm{Km}$ as the $\mathrm{X}$-Axis and Y-Axis respectively.

2. eNodeB2 is in $(500,100) \mathrm{Km}$ as the $\mathrm{X}$-Axis and $\mathrm{Y}$-Axis respectively.

3. eNodeB3 is in $(90,1500) \mathrm{Km}$ as the $\mathrm{X}$-Axis and $\mathrm{Y}$-Axis respectively.

4. eNodeB4 is in $(1000,1700) \mathrm{Km}$ as the $\mathrm{X}$-Axis and Y-Axis respectively.

5. SGW/PGW is in $(1400,800) \mathrm{Km}$ as the $\mathrm{X}$-Axis and $\mathrm{Y}$-Axis respectively.

\subsection{Simulation Scenarios}

In order to precisely determine the impact of the handover parameters, five different scenarios are developed each with different design considerations and characteristics.

A) Scenario one: the handover parameter that is studied in this scenario is threshold in regard to the A2A4-RSRQ handover algorithm. The UE with constant speed of $120 \mathrm{Km} / \mathrm{h}$ travels while it is handed over between the eNodeBs when needed. The scenario is run six times each time with a different threshold value. Thus, while the neighbor cell offset is kept constant in this scenario, the threshold values are varied as 8,10 , $20,30,31$, and $32 \mathrm{dBm}$. The throughput and number of required performed handovers are obtained as the performance metrics to determine the optimal value of the threshold parameter.

B) Scenario two: unlike the first scenario, this time the threshold is kept constant while the neighbor cell offset parameter varies as $0.25,0.5,1,2$, and $3 \mathrm{~dB}$. Thus, the scenario is run five times to determine the optimal value for neighbor cell offset parameter. The objective is to determine the optimal value of the offset handover parameter based on the performance metrics.

C) Scenario three: while the two previous scenarios investigate the A2A4-RSRQ handover algorithm, this scenario takes into account the A3-RSRP algorithm. The scenario is run 12 times to examine 12 different values for the hysteresis parameter as $0.25,0.5,1,2,3,4,5,6,7,8,9$, and $10 \mathrm{~dB}$. Thus, while the time to trigger remains constant, the optimal value for the hysteresis handover parameter is determined in terms of performance metrics.

D) Scenario four: unlike the third scenario, here the hysteresis parameter remains unchanged, and the time to trigger parameter varies as $0,40,64,80,100,128,160$, and 256 milliseconds to characterize its optimal value.

E) Scenario 5: this scenario considers the optimized value of the threshold and neighbor cell offset parameters for A2A4-RSRQ algorithm obtained in scenarios one and two respectively and also the optimized value of hysteresis and time to trigger parameters for A3-RSRP algorithm obtained in scenarios three and four respectively. Then by varying the acceleration of the car, the throughput of the A2A4-RSRQ and A3RSRP algorithms are compared under these optimized handover parameters in term of downlink throughput. The car speed varies as 30, 40, 50, 60, 70, 80, 90, 100,110, and $120 \mathrm{~km} / \mathrm{h}$.

In all scenarios, the total distance traveled by the UE is $2000 \mathrm{Km}$. the simulation time of all the scenarios depends on the speed of the UE as defined below:

Simulation time $=\frac{\text { Total distance }}{\text { Speed }}$

The design characteristics of the scenarios are summarized in Table 2.

Table2. Design characteristics of the scenarios

\begin{tabular}{ll}
\hline Name & Value \\
\hline UE speed & scenarios 1,2,3,4:120 km/h \\
& scenarios 5: $\{30,40,50,60,70,80,90,100,110,120\} \mathrm{km} / \mathrm{h}$ \\
Total distance & 2000 Km \\
Simulation time & Total distance/speed \\
Mobility algorithm & Car: ConstantVelocityMobilityModel \\
& $\begin{array}{l}\text { Four eNodeBs, SGW, PGW: ConstantPositionMobilityModel } \\
\text { scenarios 1 and 2: A2A4-RSRQ }\end{array}$ \\
Handover algorithm & scenarios 3 and 4: A3-RSRP \\
& $\{8,10,20,30,31,32\} \mathrm{dBm}$ \\
Threshold & $\{0.25,0.5,1,2,3\} \mathrm{dB}$ \\
Neighbor cell offset & $\{0.25,0.5,1,2,3,4,5,6,7,8,9,10\} \mathrm{dB}$ \\
Hysteresis & $\{0,40,64,80,100,128,160,256\}$ millisecond \\
Time to Trigger & \\
\hline
\end{tabular}




\section{SIMULATION RESULTS}

This section provides the results obtained from implementing the designed scenarios.

\subsection{Scenario1: Impact of Serving Cell Threshold}

For a constant amount of neighbor cell offset, we examine different amounts for threshold parameter to evaluate the corresponding influences on downlink throughput and number of handovers in A2A4-RSRQ algorithm. The scenario varies the threshold parameter with the purpose of finding its optimum margin. The results are presented in Figure 2.
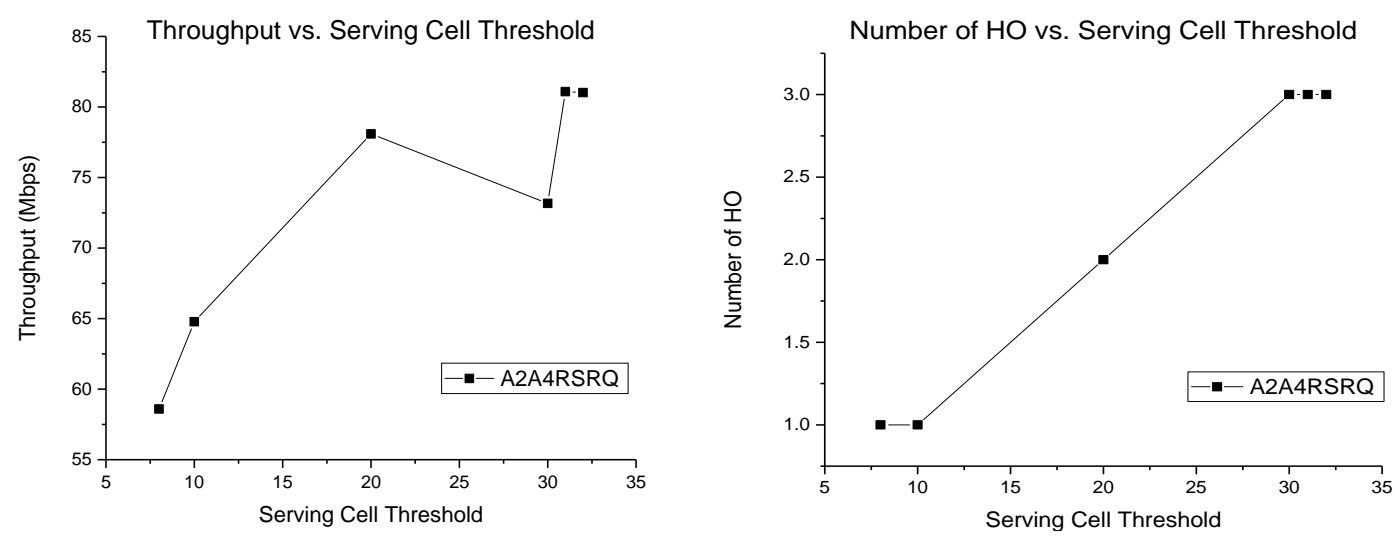

Figure 2. Impact of the threshold handover parameter on LTE performance

The results of this experiment indicate the direct effect of increasing the values of the threshold parameter on optimizing the downlink throughput. As the threshold parameter increases, the average throughput also increases accordingly as in the best point it reaches from 60Mbps to $83 \mathrm{Mbps}$. On the other hand, since there are total four eNodeBs in the scenario, the recommended maximum number of handovers will be three. As we can see, when threshold increases, the number of performed handovers also increases in parallel but does not exceed the recommended maximum number. Thereby, increasing the number of handovers does not limit the performance of the network in term of throughput. Hence the results suggest that increasing the threshold value to the highest point $(35 \mathrm{dBm})$ can accordingly enhance the downlink throughput without concern regarding to the number of unnecessary handovers.

\subsection{Scenario2: Impact of Neighbor Cell Offset}

In this scenario, we consider the influences on downlink throughput and number of handovers in A2A4-RSRQ algorithm when the neighbor cell offset parameter is variable at the same time that the threshold parameter is kept at a fixed value. The results that relate to this basis are provided in Figure 3.
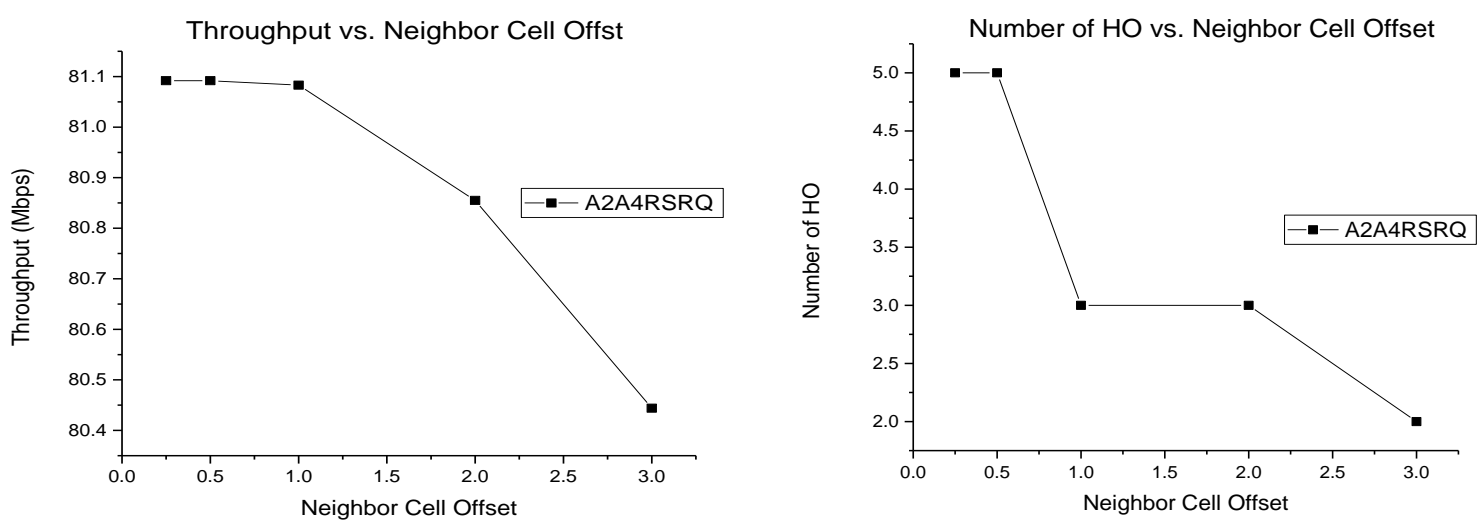

Figure 3. Impact of the neighbor cell offset handover parameter on LTE performance 
Based on the results of the experiment, the tradeoff between higher throughput alongside of increasing the number of handover is determined. It is observed that as the offset parameter increases, the average throughput is reduced while the amount of reduction is not considerable. In contrast, the higher offset value provides lower number of handovers. Thus by ignoring the low amount of throughput degradation, the larger offset parameter can provide a better network performance in term of less number of required handovers.

\subsection{Scenario3: Impact of Hysteresis}

In this scenario, we are interested in characterizing the optimal values of the hysteresis handover parameter that can affect the A3-RSRP algorithm. We consider a fixed amount for time to trigger parameter while varying the amount of hysteresis to evaluate the effects on downlink throughput and number of handovers. The results are illustrated in Figure 4.
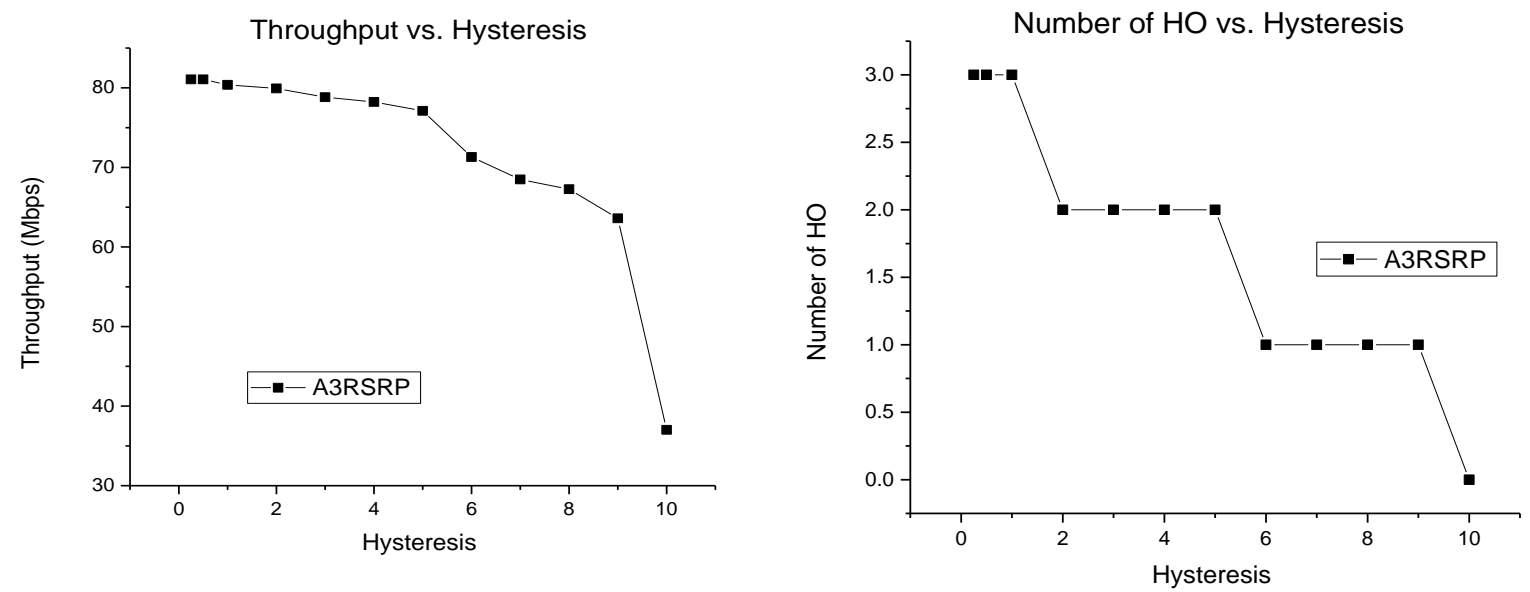

Figure 4. Impact of the hysteresis handover parameter on LTE performance

The fact that the slope declines as the hysteresis is increased illustrates the effectiveness of the hysteresis parameter on reduction of the throughput. As the hysteresis increases, the throughput decreases so that the lowest throughput is achieved under the highest amount of hysteresis. The throughput degrades dramatically from $80 \mathrm{Mbps}$ to $35 \mathrm{Mbps}$ when the hysteresis reaches its highest value $(10 \mathrm{~dB})$. Also under the highest value of the hysteresis, the number of performed handovers reaches its lowest value. However, since still the highest number of handovers does not exceed the recommended maximum value (3), we can say that under the lowest hysteresis value, the highest throughput with an acceptable number of handovers are achieved. Thus the lower the hysteresis values can provide better performance for LTE networks.

\subsection{Scenario4: Impact of Time to Trigger}

This scenario assigns a constant level for the hysteresis in A3-RSRP algorithm while varying the time to trigger parameter. Thus by assigning different time slots to the time to trigger handover parameters, we determine the results of the corresponding impacts on the downlink throughput which are presented in Figure 5.

As the results illustrate, increasing the time to trigger parameter will decrease the average of downlink throughput. Based on the results, the optimized value of time to trigger handover parameter is obtained at $50 \mathrm{~ms}$ achieving the highest average throughput. 


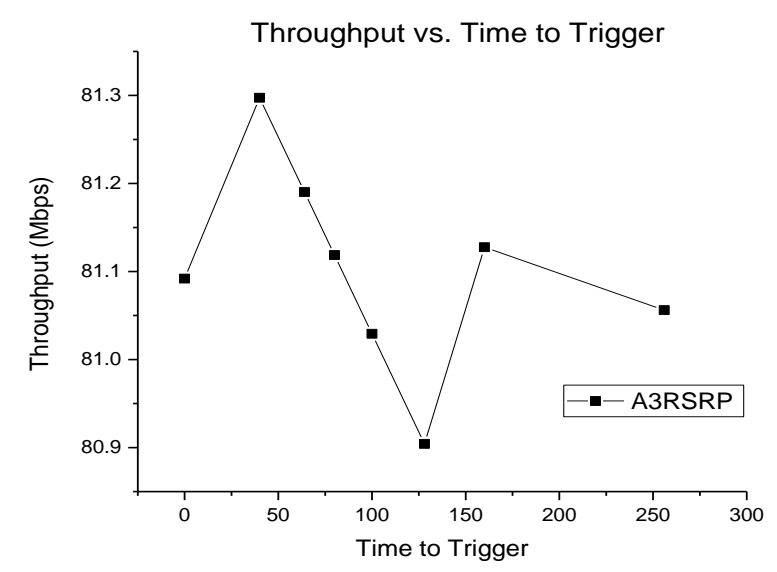

Figure 5. Impact of the time to trigger handover parameter on LTE performance

\subsection{Scenario5: Impact of the Optimized Handover Parameters}

From implementing the four previous scenarios, we determined the optimal values for the four handover parameters involved in both handover algorithms. Here, we use these optimal values to compare the performance of A2A4-RSRQ and A3-RSRP handover algorithms under the obtained optimized handover parameters in term of downlink throughput. The results are provided in Figure 6.

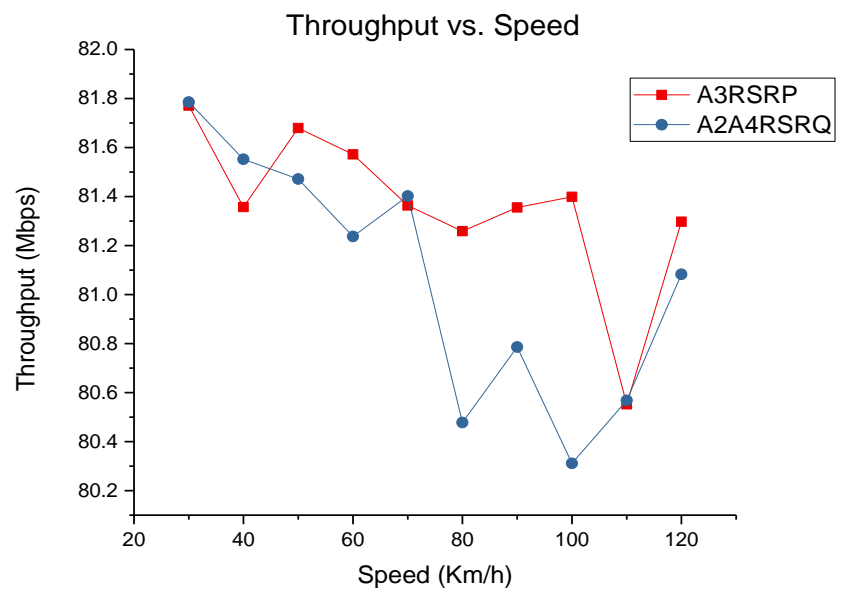

Figure 6. Impact of the optimized parameters on LTE performance

This results mainly take into account the performance comparison of the A3-RSRP and A2A4RSRQ handover algorithms under different speeds of the UE. The results show higher throughput for the A3RSRP algorithm under the optimized handover parameters. As the speed increases, the downlink throughput slightly decreases in both algorithms while still the A3-RSRP algorithm achieves higher downlink throughput.

\section{CONCLUSION}

In this work we investigated the four main handover parameters in the two current handover algorithms. The optimal values for the parameters obtained to optimize the overall handover process which directly affects the LTE performance. Then the obtained optimal values were applied over the two handover algorithms to furthermore compare their performance under optimal conditions. Our results proved that varying any of the handover parameters, can highly affect the overall LTE performance. Thus, pre-selecting of the proper values is a necessary requirement in the early stages before any handover decision is made. 


\section{REFERENCES}

[1] L. Zhang, T. Okamawari, and T. Fujii, Performance Evaluation of TCP and UDP during LTE Handover, IEEE Wireless Communications and Networking Conference: Mobile and Wireless Networks, pp.1993-1997, 2012.

[2] N. Shojaedin, M. Ghaderi, and A. Sridharan, Effect of Handover on the Performance of Scheduling Algorithms in LTE Networks, IEEE Wireless Communications and Networking Conference (WCNC), pp.1386-1391, 2015.

[3] R. Tao, L. Li, X. Chu, and J. Zhang, Handover Mechanism and Performance Evaluation for LTE-LAA systems, IEEE 17th International Workshop on Signal Processing Advances in Wireless Communications (SPAWC), pp.1-5, 2016.

[4] Z. Guohua, P. Legg, and G. Hui, A Network Controlled Handover Mechanism and its Optimization in LTE Heterogeneous Networks, IEEE Wireless Communications and Networking Conference (WCNC), pp.1915-1919, 2013.

[5] L. Luan, M. Wu, Y. Chen, X. He, and C Zhang, Handover parameter optimization of LTE system in variational velocity environment, IET International Conference on Communication Technology and Application (ICCTA), pp.1-5, 2011.

[6] 3GPP, "Evolved Universal Terrestrial Radio Access (E-UTRA); Physical layer; Measurements," in TS 36.214, ed. France: Sophia Antipolis, 2012.

[7] D. V. Zavyalova, M. L. Rolich, A. V. Andreev, Definition the optimal parameters of handover procedures in LTE networks, IEEE 17th International Conference of Young Specialists on Micro/Nanotechnologies and Electron Devices (EDM), pp.110-113, 2016.

[8] T. Komine, T. Yamamoto, and S. Konishi, A Proposal of Cell Selection Algorithm for LTE Handover Optimization, IEEE Symposium on Computers and Communications (ISCC), pp.37-42, 2012.

[9] R. Prasad Ray and L. Tang, Hysteresis Margin and Load Balancing for Handover in Heterogeneous Network, International Journal of Future Computer and Communication, Vol.4, No.4, 2015.

[10] H. D. Bae, B. Ryu, and N. Park, Analysis of Handover Failures in LTE Femtocell Systems, Australasian Telecommunication Networks and Applications Conference (ATNAC), pp.1-5, 2011.

[11] N. Katti, S. Shivapur, and M. Vijayalakshmi, Optimization of QoS in 4G Networks Using Handover Management International Journal of Emerging Technology in Computer Science \& Electronics (IJETCSE), Vol.14, No.2, 2015.

[12] C. C. Lin, K. Sandrasegaran, H. A. M. Ramli, and R. Basukala, optimized performance evaluation of LTE hard handover algorithm with average RSRP constraint, International Journal of Wireless \& Mobile Networks (IJWMN) Vol.3, No.2, 2011.

[13] F. Afroz, R. Subramanian, R. Heidary, K. Sandrasegaran and S. Ahmed, SINR, RSRP, RSSI AND RSRQ measurements in long term evolution networks, International Journal of Wireless \& Mobile Networks (IJWMN), Vol.7, No.4, 2015.

[14] Y. Zhang, M. Wu, S. Ge, L. Luan, and A. Zhang, Optimization of time-to-trigger parameter on handover performance in LTE high-speed railway networks, 15th International Symposium on Wireless Personal Multimedia Communications (WPMC), pp.251-255, 2012.

[15] K. Alexandris, N. Nikaein, R. Knopp, and C. Bonnet, Analyzing X2 Handover in LTE/LTE-A, 14th International Symposium on Modeling and Optimization in Mobile, Ad Hoc, and Wireless Networks (WiOpt), pp.1-7, 2016.

[16] A. S. Priyadharshini and P. T. V. Bhuvaneswari, A Study on Handover Parameter Optimization in LTE-A Networks, International Conference on Microelectronics, Computing and Communications (MicroCom), pp.1-5, 2016.

[17] B. V. Arun and D. Jayaramaiah, Performance Evaluation of LTE Hard Handover Algorithm with Multimedia Data Transmission, International Journal of Innovative Research in Computer and Communication Engineering, Vol.2, No.4, 2014.

[18] M. Mehta, N. Akhtar, and A. Karandikar, Impact of HandOver Parameters on Mobility Performance in LTE HetNets, Twenty First National Conference on Communications (NCC), pp.1-6, 2015.

[19] R. Zhang, M. Wu, and Y. Zhang, Analysis of Handover Trigger Scheme Based on Distance for LTE High-speed Railway Networks, TELKOMNIKA (Telecommunication, Computing, Electronics and Control), Vol.14, No.1, pp. 129-135, 2016.

[20] I. Hidyat, SC-FDMA LTE Performance through High Altitude Platforms Communications (HAPS) Channel, TELKOMNIKA (Telecommunication, Computing, Electronics and Control) Vol.14, No.2, pp.515-522, 2016.

[21] I. Galih, Carrier Aggregation Technique to Improve Capacity in LTE-Advanced Network, TELKOMNIKA (Telecommunication, Computing, Electronics and Control), Vol. 14, No. 1, pp.119-128, 2016 


\section{BIOGRAPHY OF AUTHORS}

Mina Malekzadeh is an assistant professor and lecturer in the department of computer science at Hakim Sabzevari University. Her research interests include communication networks, network security, VoIP, and system development programming. She holds a Doctoral degree in computer security from UPM, MSc in software engineering from UPM.

Fatemeh Rezaiee received the B.S. degree in Computer Science from Hakim Sabzevari University. She is currently a master candidate in software engineering. Her research interests are Computer Networks and wireless security. 